Supplement of The Cryosphere Discuss., 9, 1-44, 2015

http://www.the-cryosphere-discuss.net/9/1/2015/

doi:10.5194/tcd-9-1-2015-supplement

(C) Author(s) 2015. CC Attribution 3.0 License.

(c) (i)

Supplement of

\title{
Theoretical framework for estimating snow distribution through point measurements
}

\author{
E. Trujillo and M. Lehning
}

Correspondence to: E. Trujillo (ernesto.trujillo@epfl.ch) 


\section{Supplement I: Relevance of the terms in the expression to determine the normalized}

squared error

The survey design in Figure 14a (Main Article) can be used to illustrate the roll of each of the terms in the equation for determining the optimal location of the points. The objective is to find the value of $a$ that for a given area minimizes $\sigma_{\bar{Z}}^{2}$. Such analysis requires knowledge of the correlation structure, fully described by the parameters of the correlation function ( $v$ for the exponential model).

In the analysis that follows, the terms are normalized by the variance $\sigma_{p}^{2}$. The first term is the inverse of the number of points, which takes a value of 0.2 for this example. With $N$ fixed at 5 , the value of $L$ can be fixed such that the value of the second term can be determined for several values of the decay exponent (v), and for $a$ varying between 0 and $L / 2$. In the example, $L$ is set to 30, and $v$ is varied between 0.1 and 5. The resulting curves for terms 2 and 3 are shown in Figure S 1a (positive curves for term 2 and negatives for term 3), and the sum of the two terms for shown in Figure $\mathrm{S} 1 \mathrm{~b}$. The value of the second term decays as $a$ increases, reaching the minimum value when the outer points are located at the boundaries of the domain. This result is logical, the farther the distance between the measurements the lesser the correlations. This second term is driving the location of the points towards the boundary of the domain; however, if the correlation decays fast enough, a value close to the minimum can be reached at a distance shorter than L/2 (e.g., see black curves in Figure S 1a). On the other hand, term 3 takes a maximum absolute value when all the points are located at the center of the area (i.e., $a=0$ ), and decreases its absolute value as $a$ increases. The effect of this third term counters the effect of term 2, and drives the points closer to the center of the area; however, as $v$ increases, the value of 
term 3 decreases and becomes almost irrelevant (e.g., see term 3 curves for $v>1$ in black). The sum of terms 2 and 3 determine the value of $a$ that minimizes $\sigma_{\bar{Z}}^{2}$ for a given $v$ (Figure $\mathrm{S} 1 \mathrm{~b}$ ). A general conclusion is that the optimal value of $a$ that minimizes $\sigma_{\bar{Z}}^{2}$ decreases as $v$ increases, and as the value of term 3 becomes insignificant, an effective minimum can be reached very close to the center (e.g., Figure $\mathrm{S} 1 \mathrm{~b}$ for $v>1$ ).

(a)

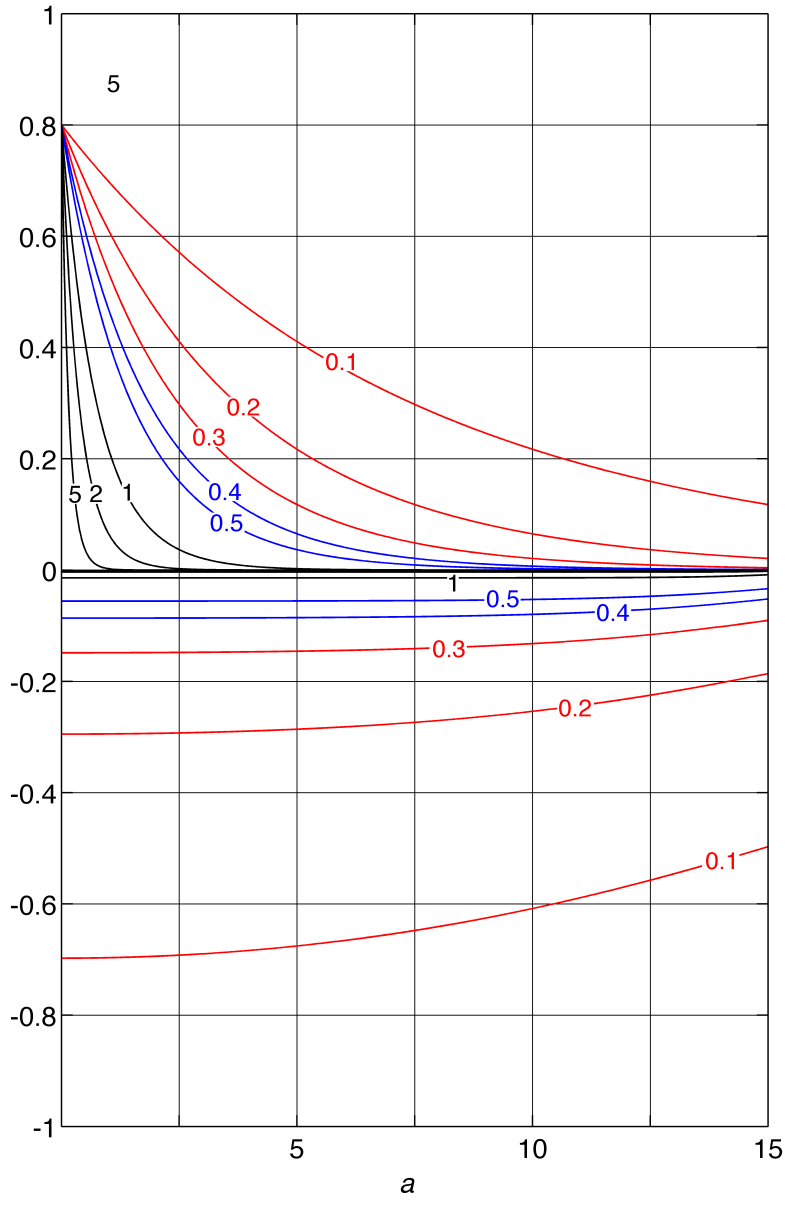

(b)

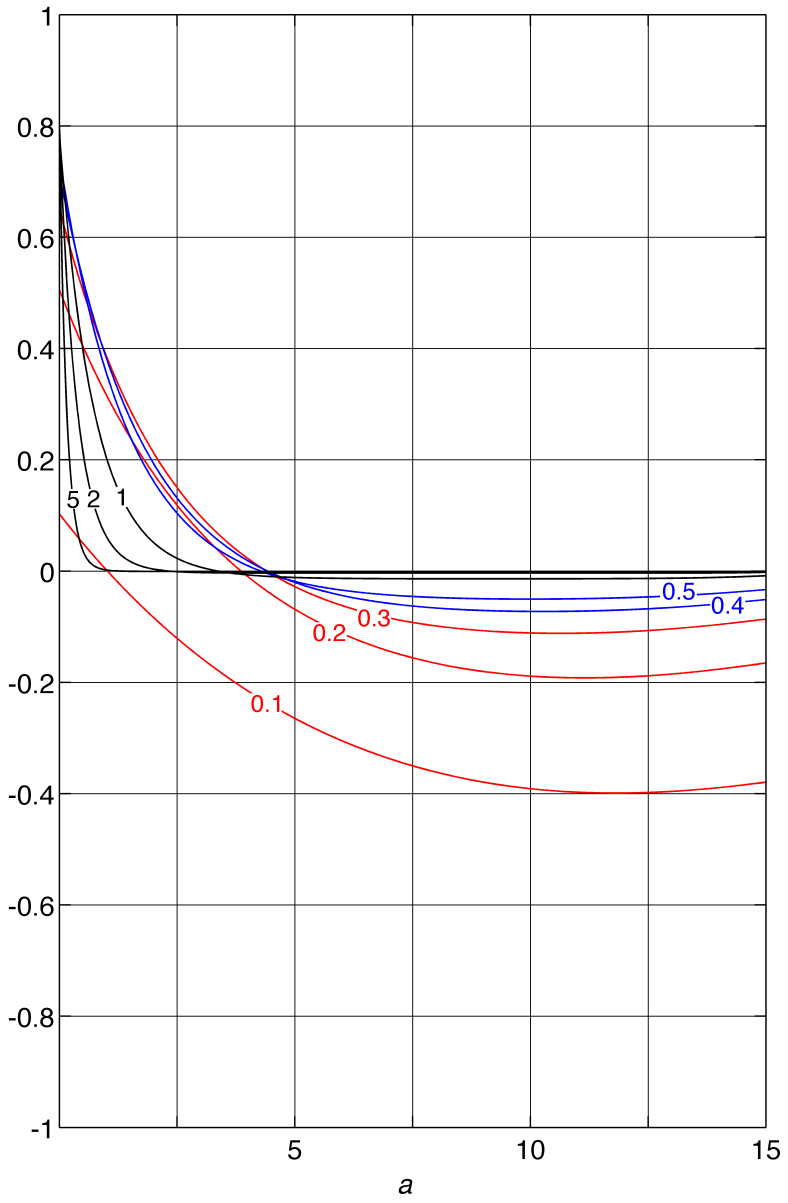

Figure S 1. (a) Term 2 (positive curves) and Term 3 (negative curves) as a function of $a$ for decay exponent values ( $v$ ) between 0.1 and 5. (b) Sum of Term 2 and Term 3 as a function of $a$ for decay exponent values $(v)$ between 0.1 and 5 . Colors are used for identification purposes. 
As discussed above, term 4 is independent of the location of the points and is only a function of the domain (shape and area) and the correlation structure (e.g., v). The value of term 4 as a function of $v$ for an area of side dimension 30 is shown in Figure S 2. Similar to what is seen for tem 3 , the value of term 4 decays with $v$, reaching values below 0.01 for $v>1$. The value that term 4 using the exponential model for areas of side dimension between 1 and 100 is shown in Figure $\mathrm{S} 3$. The value of term 4 is higher as the area decreases, since the term is inversely proportional to the square of the area size, and the correlation value is highest for shorter lags.

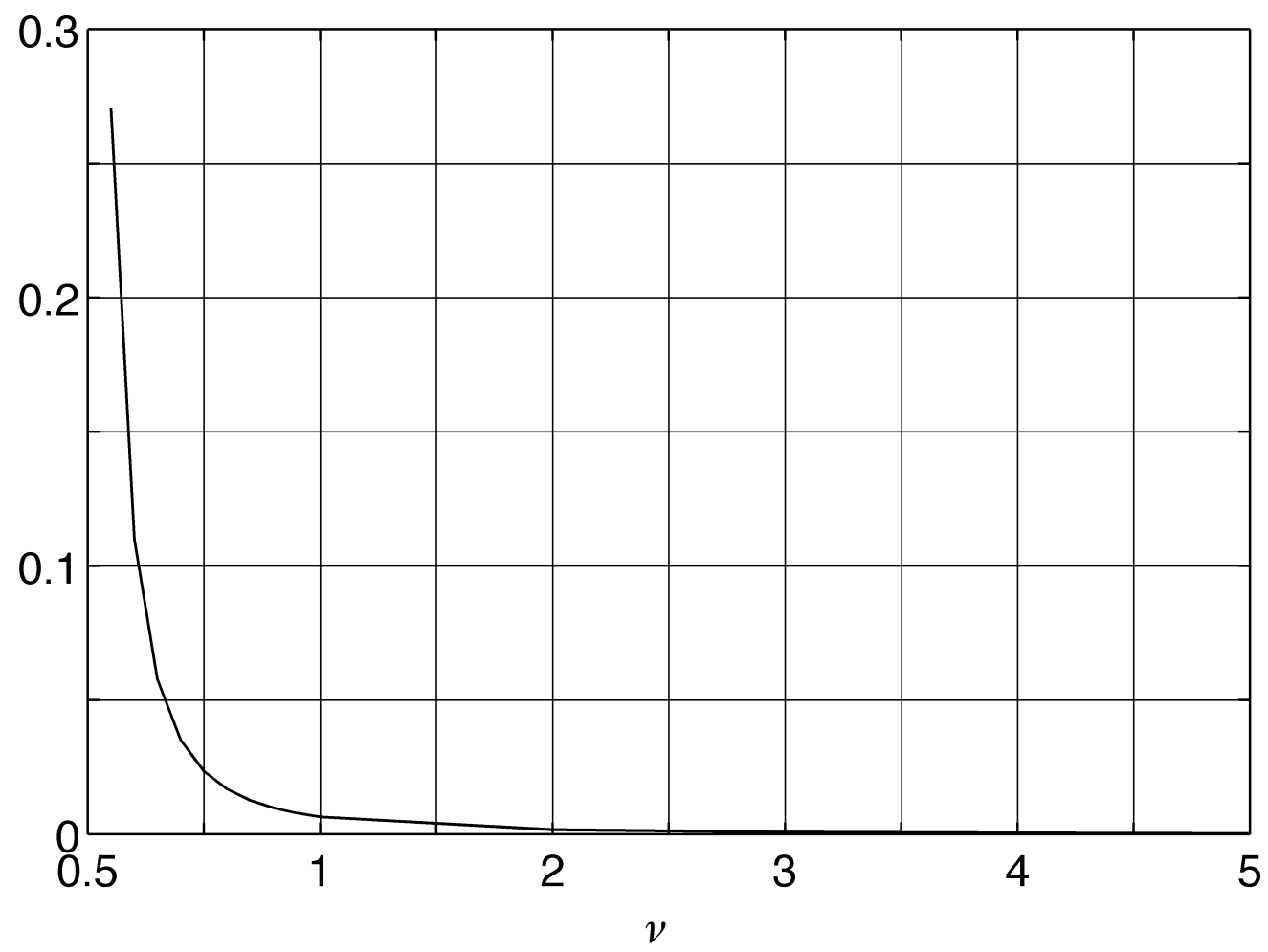

Figure S 2. Term 4 as a function of $v$ for an area of side dimension of 30 . 


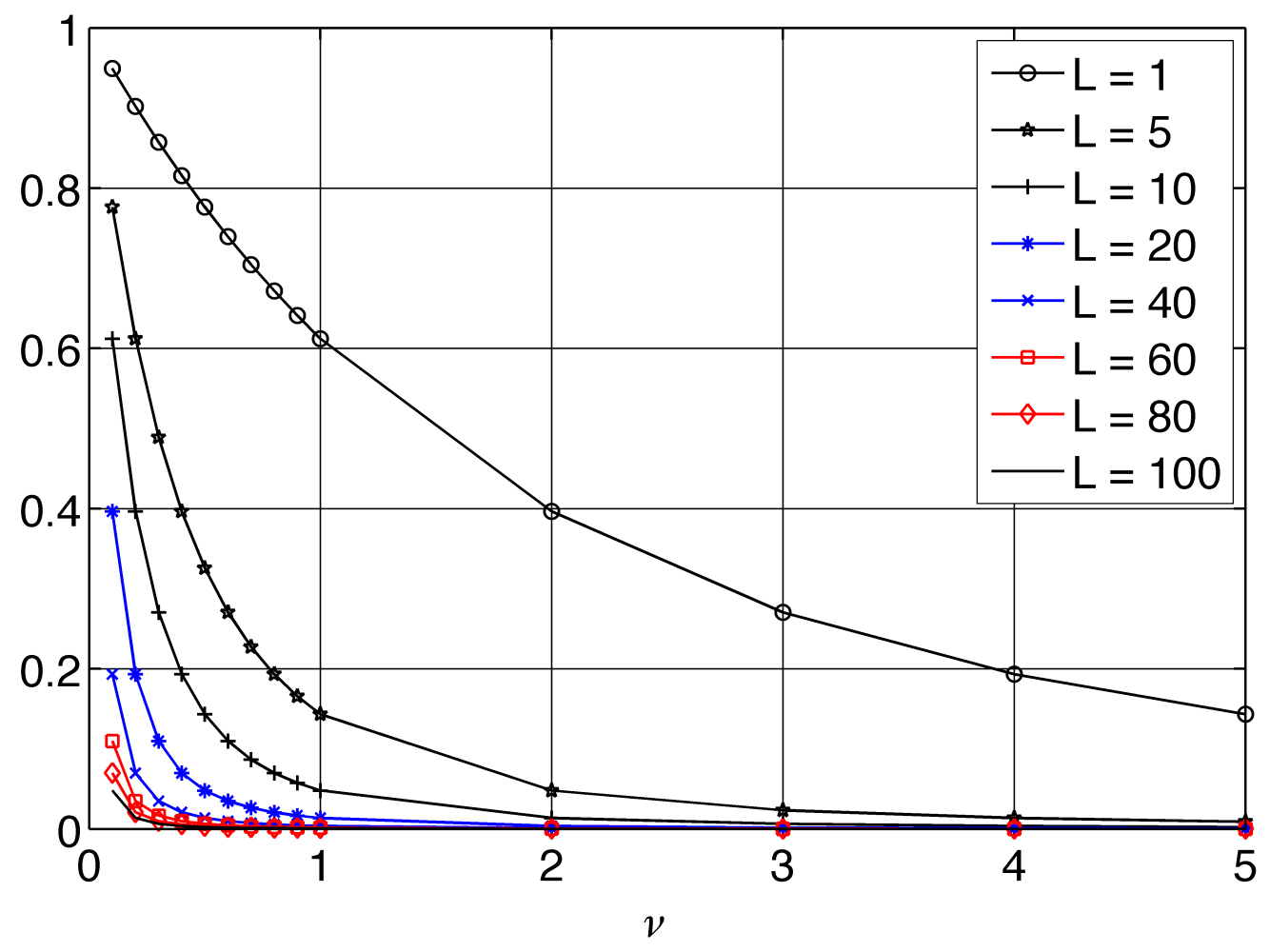

Figure S 3. Term 4 as a function of $v$ for areas of side dimension between 1 to 100 . 